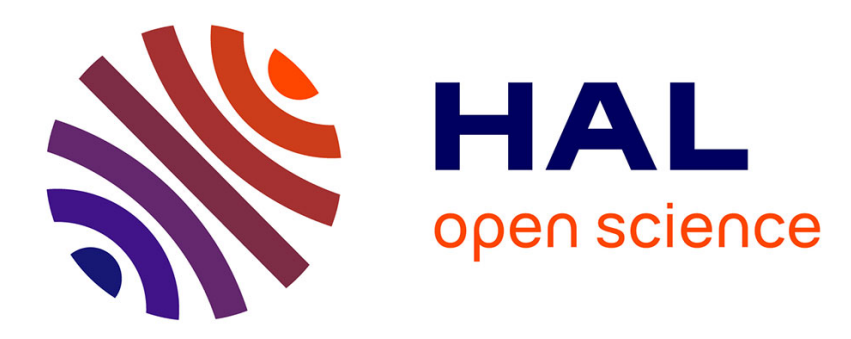

\title{
LES INJECTIONS THÉRAPEUTIQUES DE LAIT EN MEDECINE VÉTÉRINAIRE
}

\author{
L. Panisset, J. Verge
}

\section{To cite this version:}

L. Panisset, J. Verge. LES INJECTIONS THÉRAPEUTIQUES DE LAIT EN MEDECINE VÉTÉRINAIRE. Le Lait, 1922, 2 (6), pp.417-428. hal-00894703

\section{HAL Id: hal-00894703 \\ https://hal.science/hal-00894703}

Submitted on 1 Jan 1922

HAL is a multi-disciplinary open access archive for the deposit and dissemination of scientific research documents, whether they are published or not. The documents may come from teaching and research institutions in France or abroad, or from public or private research centers.
L'archive ouverte pluridisciplinaire HAL, est destinée au dépôt et à la diffusion de documents scientifiques de niveau recherche, publiés ou non, émanant des établissements d'enseignement et de recherche français ou étrangers, des laboratoires publics ou privés. 
pondéral. D'autre part, certaines intolérances peuvent, dès le début, se manifester par une stagnation de la courbe de poids alors que les vomissements font défaut et ne surviennent que plusieurs semaines après. Enfin, il est possible de constater à la suite des injections de lait une amélioration de l'état général et une reprise de poids alors que les vomissements persistent quelques jours encore. Ces constatations permettent de dissocier les facteurs pathogéniques de l'hypotrophie; et tout en accordant une part importante à l'inanition, aux troubles digestifs et nerveux, à l'insuffisance alimentaire, de faire intervenir un trouble nutritif dépendant de l'action toxique qui est à la base de l'intolérance.

(A suivre).

\title{
LES INJEGTIONS THERAPEUTIQUES DE LAIT EN MEDEGINE VETERINAIRE
}

\author{
par \\ L. PANISSET, et J. VERGE, \\ Professeur Chef de Travaux \\ à l'Ecole vétérinaire d'Alfort.
}

La valeur thérapeutique des injections de lait est discutée dans les milieux scientifiques. Certains auteurs estiment que le procédé réalise un grand progrès de thérapeutique générale; entre les mains d'autres chercheurs, les injections de lait,n'ont apporté aucun bénéfice sérieux dans la cure des maladies.

Par des essais expérimentaux chez le cobaye, par des tentatives de traitement de maladies diverses chez les bovidés et le chien, nous nous sommes fait une opinion sur le champ d'action et la valeur de cette nouvelle médication.

C'est cette opinion que nous apportons ici.

Avant d'aborder la question du point de vue expérimental, nous voudrions mettre en parallèle les résultats déjà acquis sur la valeur thérapeutique des injections de lait. De suite, nous sommes obligés de constater que les travaux des savants diffèrent en la matière et que, sur certains points même, leurs conclusions șont diamétralement 
opposées. Peut-être cela tient-il à ce que le produit utilisé n'est pas le même partout.

En Allemagne, en particulier, si on a employé le lait en nature lait stérilisé au préalable - il semble quel'on ait donné la préférence à certaines préparations fournies par le commerce sous le nom d'aolan, de lactosan, d'albijon, d'ergolactine, d'eugalactan, de caséosan, etc..., ces préparations multiples renferment, les unes les albumines du lait, d'autres, la caséine; elles présentent ce caractère commun que leur introduction dans l'organisme se fait par une voie parentérale: intra-veineuse, intra-musculaire, sous-cutanée; la voie intra-musculaire étant la plus souvent utilisée.

Chez l'homme, de nombreuses affections ont semblé justiciables de cette thérapeutique; c'est ainsi que le Professeur Wertl, de Lyon, et, après lui, le Docteur Temporal en font « le traitement de choix de l'intolérance du nourrisson pour le lait ». C'est ici une méthode d'antianaphylaxie, de désensibilisation spécifique.

Dans les affections inflammatoires des yeux, de nombreux médecins (Jocos, Darier, Domec, Marie Amat) ont obtenu d'excellents résultats. De même, dans le traitement de diverses maladies infectieuses de l'enfant ou de l'adulte: grippe et ses complications (Thiroloix, Jocos, Joltrain, Aмat), fièvre typhoïde (Sснмidt, MüLLER), typhus exanthématique (SAxL), orchite et salpingite blennorragiques (K. AdAM, WEISs, MÜLLER), fièvre puerpérale, anémie pernicieuse (SснміDт), etc. Certaines affections parasitaires ou cutanées sont notablement améliorées par les injections de lait (Schmidt, Kagullson, Müller, Weichard, Leiner, Ambroseli).

Par contre, dans le cancer, la chlorose, la paralysie générale, le lait n'aurait apporté aucun changement dans l'état des malades (Sghmdt, Kagullson).

En médecine vétérinaire, le lait a déjà été très employé. Il ne semble pas cependant que les vétérinaires français aient eu recours à ce moyen de traitement. Les périodiques scientifiques suisses et allemands offrent au contraire nombre d'observations presque toutes favorables à l'emploi thérapeutique du lait dans la lutte contre de multiples affections et les maladies contagieuses des animaux domestiques.

On signale l'efficacité du produit dans la fièvre aphteuse des bovidés (Müller, Thun, etc.), la fièvre aphteuse du porc (Müller), le coryza gangréneux (Grawert, Rieger, Fischer), les abcès, les 
phlegmons aigus ou chroniques, l'actinomycose (RIEGER), la botryomycose (Grawert, Fröhlich), l'eczéma et la lymphadénie deschiens. Les mammites des vaches laitières, l'arthrite des poulains, au moins dans les vingt-quatre heures qui suivent la naissance, le rouget (Müller, Schmaling, Berndt), la maladie du jeune âge chez le chien tireraient un grand profit des injections sous-cutanées ou intra-musculaires de lait.

Les maladies cutanées, parasitaires ou non, sont justiciables de la même thérapeutique. D'excellents résultats sont obtenus dans le traitement des différentes formes, aigües ou chroniques, d'eczéma ou d'acné, dans la furonculose, la nécrobacillose, l'herpès, le favus, etc. (Grawert, Fröhlich).

En ce qui concerne la fièvre aphteuse, il est intéressant de relever en détail les observations : 24 beures après l'injection médicamenteuse, l'état général s'améliore notablement et la production lactée de la mamelle remonte dès le $4^{\mathrm{e}}$ jour (1). Les aphtes des onglons guérissent rapidement, ceux de la langue et dela bouches'ouvrent toujours 24 heures après l'injection. Des altérations traitées seulement quelques jours après leur apparition, guérissent très vite et une épidermisation intense vient réparer alors ces brèches cutanées, en particulier au niveau des ulcères de la langue.

Mais, en poursuivant le dépouillement des publications consacrées à la méthode, on constate que de nombreux insuccès ont été mentionnés: actinomycose (Herss), lymphangite épizootique (Grawert, EISENBLÄTtER), certaines ophtalmies, l'entérite chronique.

Des cas de rouget (MüLLER), de botryomycose, de coryza gangréneux (Rigger, Heiss) se seraient montrés iebelles. Enfin, lors d'une invasion de rouget, Thомson estime même que les injections de lait n'auraient servi qu'à affaiblir les porcs ainsi traités et à les rendre plus réceptifs à l'infection.

Quelles sont les modes d'introduction du lait dans les organismes animaux ? Différents moyens ont été proposés : inoculation souscutanée, intra-veineuse ou intra-musculaire. Il semble que l'injection dans le muscle soit celle qui donne les meilleurs résultats et qui mette en même temps à l'abri des phénomèmes d'anaphylaxiesuscep-

(1) L'action galactogène des injections sous-cutanées de lait chez la vache, est bien connue. 
tibles de se manifester en quelques cas (Lubliner). Pour notre part, c'est toujours par voie intra-musculaire que nous avons injecté les solutions de lait, aussi bien chez le bœuf que chez le chien et le cobaye.

La dose varie, c'est évident, avec l'espèce animale envisagée.

Chez le cheval et le bouf, on peut injecter en une seule fois, dans les masses musculaires de la croupe ou de la fesse, 50 à $100 \mathrm{~cm}^{3}$ de lait. La résorption se fait parfaitement, sans laisser de traces, dans les heures qui suivent: nul engorgement, nulle induration ne sont à redouter.

Chez le veau, le mouton, la chèvre, la dose à injecter en une seule fois est de $25 \mathrm{~cm}^{3}$. Chez le pore, la quantité est proportionnelle au poids des animaux; dans la règle, de 10 à $25 \mathrm{~cm}^{3}$ sont nécessaires.

Le chien recevra, dans les muscles de la fesse, 5 à $10 \mathrm{~cm}^{3}$ de lait stérile ou d'une solution stérile de lactalbumine; le chat, $3 \grave{a} 5 \mathrm{~cm}^{3}$ seulement.

De plus, sans crainte de provoquer des phénomènes toujours dangereux et quelquefois mortels d'hypersensibilité, on peut réinjecter les animaux ainsi traités, aux mêmes doses, 3 à 4 jours après l'injection primitive. En certains cas même, il est loisible au praticien de répéter chaque jour l'inoculation, sans que l'organisme traduise dans la suite la plus légère anaphylaxie.

Ce qu'il est nécessaire surtout de savoir, c'est que le résultat doit ètre acquis en 5 à 6 injectioas. "Il ne paraît pas utile d'en faire davantage, car on se trouve alors en présence d'affections rebelles à ce traitement. ) (TemporaL).

En résumé - et c'est là le mode opératoire que nous avons adopté - s'il s'agit d'une maladie à évolution rapide, infectieuse ou non, on se Irouvera bien de l'injection quotidienne, voir même biquotidienne, de lait.

Au contraire, en présence d'une affection à marche plus lente ou d'une maladie chronique, il suffit de procéder tous les 3 ou 4 jours à l'injection intra-musculaire de lait, dans la forme prescrite.

Comment agissent le lait ou ses dérivés : lactalbumine, caséine, etc.... dans ce procédé thérapeutique? Est-ce une méthode de désensibilisation strictement spécifiqne, ou bien, plus simplement, ne faut-il voir là qu'une application particulière d'une méthode beaucoup plus générale et non étroitement spécifique: la protéinothérapie? 
L'efficacité des injections de lait dans l'intolérance des nourrissons pour cet aliment indiquerait qu'il se produit, en ce cas, une véritable désensibilisation : l'état d'intolérance disparaît alors de façon durable.

Mais, dans la plupart des cas où les injections de lait sont employées et se sont montrées efficaces, il s'agit d'autre chose. Un processus différent est en cause : le lait agit par les protéines qu'il renferme, par ses albumines hétérogènes qui vont produire des modifications certaines, non éphémères dans l'économie.

Un premier point, sur lequel ne plane aucune incertitude, c'est que le nombre des leucocytes augmente quelques heures après l'injection, augmentatation légère, il est vrai, mais qui n'est pas moins réelle (Grawert, Fröhlich, etc.). Sur un cheval sain, l'injection intra-musculaire de lait produit une hyperleucocytose manifeste : 18.000 leucocytes par millimètre cube de sang après 8 heures, 27.000 environ après 3 jours et retour au chiffre normal vers le sixième jour.

II en est de même chez le chien où une injection intra-musculaire de $10 \mathrm{~cm}^{3}$ de lait porte, en 24 heures, le chiffre des leucocytes de 10.000 à 18.000 par $\mathrm{mm}^{3}$ de sang. Les cellules blanches agiraient et par leur pouvoir phagocytaire et surtout par les ferments lytiques qu'elles produisent, protéases, en particulier, dont l'action sur les toxines issues des bactéries est manifeste (JoBling et BuLL).

De plus, à la lumière de nombreuses expériences (IснікаWA; Müller et Weiss, Jobling et Dunklin), on sait que l'injection de protéines dans les organismes malades ou immunisés augmente la proportion des anticorps que renferme le sang circulant. Le lait agit donc par une action semblable et ce renforcement de l'immunité humorale ne peut qu'être favorable à une issue heureuse des affections traitées.

Peut-être aussi, la «galactothérapie » constitue-t-elle une modalité du traitement par le choc colloïdoclasique; plus succinctement et selon les conceptions du Professeur Widal et de ses élèves, la thérapeutique par le lait ne serait qu'un chapitre étroit de la colloïdoclasothérapie.

Le lait injecté dans l'organisme par voie parentérale met en jeu des réactions d'ordre colloïdal, réactions qui s'opèrent au sein des plasmas : parfois, c'est un trouble, une rupture de l'équilibre colloïdal qui en est la conséquence, lequel trouble, comme l'a 
montré GowaErTs, modifie les réactions physiques de contact qui régissent les interactions microbes-colloïdes plasmatiques; parfois, au contraire, c'est la transformation d'une instabilité, d'un désèquilibre plasmatique préexistant avec retour vers la stabilite normale et guérison consécutive qui se produit; parfois, enfin, on assiste à la production d'un choc salutaire (thérapeutique par le choc). En ce dernier cas, Widal et ses collaborateurs ont montré que le choc protéique, dû à l'injection de lait, s'accompagne fréquemment d'une crise hémoclasique évidente, bien qu'atténuée.

En résumé, il nous semble que les injections de lait agissent différemment selon les maladies auxquelles on les oppose. C'est à un mécanisme de désensibilisation spécifique (intolérance pour le lait chez les nourrissons) ou de désensibilisation paraspécifique (maladies cutanées, eczémas, acné, etc.) qu'il faut rapporter les succès enregistrés par certains auteurs. Enfin, c'est à une excitation non spécifique des moyens généraux - humoraux et cellulaires - de défense de l'organisme que tend l'action bienfaisante du lait dans la lutte contre certains processus généraux infectieux.

Un essai d'orientation thérapeutique chez le chien nous a permis de faire quelques constatations intéressantes.

Nous n’avons employé, dans toutes nos expériences, que du lait préalablement écrémé et stérilisé à l'autoclave.

Nous avons injecté chaque jour, du 15 au 22 décembre dernier, dans les muscles de la cuisse d'un chien sain pesant $20 \mathrm{~kg}$, $10 \mathrm{~cm}^{3}$ de lait. Les injections ont toujours été très bien supportées, quoiqu'elles fussent faites toutes à peu près au même niveau. Nous n'eùmes à enregistrer ni infiltration locale, ni œdème, ni abcédation consécutive; la démarche ne fut jamais gênée et les muscles du lieu de l'injection n'ont paru à aucun moment sensibles et douloureux à la pression.

Les phénomènes généraux ont été strictement limités : nous n'avons pas constaté de fièvre dans les heures qui suivirent l'inoculation intra-musculaire ; nous n'avons noté aucun symptôme de choc protéique ou de sensibilité anaphylactique à l'occasion de la répétition des injections. Seul, un amaigrissement de $3 \mathrm{~kg}$. traduisait, en fin d'expérience, les réactions intimes de l'organisme ainsi sollicité.

Chez l'homme cependant, LUBLINER, contrairement aux assertions 
d'autres auteurs, note une hyperthermie constante après l'injection et propose même pour cette fievre le nom de «maladie du laiı » par analogie avec la maladie du sérum.

Nous avons expérimenté la valeur des injections de lait dans deux maladies artificiellement provoquées : fièvre charbonneuse chez le cobaye, rouget chez la souris.

\section{Action du lait lans la fièvre charbonneuse expérimentale} du cobaye. - La fièvre charbonneuse expérimentale était déterminée par inoculation d'une culture de 24 heures en bouillon peptoné, de la bactéridie ; inoculation pratiquée sous la peau avec la dose minima mortelle que des essais nous avait montré être d'un cent millième de centimètre cube. Cetle dose tuait le cobaye en 48 heures environ.

Le protocole de chacune de nos expériences était ainsi fixé : 2 cobayes, A et B, inoculés sous la peau avec la dose minima de culture charbonneuse servaient de témoins:

2 cobayes $G$ et $D$ recevaient, au préalable, 5 jours durant, une injection intra-musculaire d'un centimètre cube de lait ; le $6^{\mathrm{e}}$ jour, on leur insérait, sous la peau, $\frac{1}{100.000}$ de $\mathrm{cm}^{3}$ de la culture charbonneuse de 24 heures (traitement préventif);

2 cobayes $\mathrm{E}$ et $\mathrm{F}$ étaient inoculés dans les muscles de la cuisse avec le mélange suivant, pratiqué dans la seringue même : $1 \mathrm{~cm}^{3} \mathrm{de}$ lait stérile $+\frac{1}{100.000} \mathrm{~cm}^{3}$ de la culture virulente (méthode simultanée par mélange);

2 cobayes $\mathrm{G}$ et $\mathrm{H}$ recevaient au même moment, mais en des points différents du corps, $2 \mathrm{~cm}^{3}$ de lait par voie intra-musculaire et $\frac{1}{100.000} \mathrm{~cm}^{3}$ de la culture par voie sous-cutanée (méthode simultanée).

2 cobayes I et $K$, injectés sous la peau avec la dose mortelle de bactéridie, recevaient de 8 heures en 8 heures, à partir de la $12^{\mathrm{e}}$ heure suivant l'inoculation virulente, des injections intra-musculaires de lait (traitement curatif);

Enfin 2 cobayes $L$ et $M$, inoculés simultanément à l'instar de leurs congénères $\mathrm{G}$ et $\mathrm{H}$, recevaient ensuite et dans les mêmes formes, le traitement appliqué aux cobayes I et K (traitement símultané et curatif). 
Cette expérience fut instituée à 5 reprises différentes. II est à noter que, parfois, l'injection intra-musculaire de lait dans la cuisse du cobaye déclenche un œè̀me léger, diffus, pouvant même s'étendre sur la paroi abdominale inférieure.

Les résultats ne nous permettent guère de conclure à l'efficacité de la médication lactée telle que nous l'avons expérimentée dans le charbon expérimental du cobaye. Seules seraient à retenir, en faveur de la méthode, deux survies de 24 heures chez deux cobayes ayant reçu, lors de notre troisième expérience, l'un des injections de lait à titre curatif, l'autre des injections de lait en même temps que le virus d'abord, puis à titre curatif ensuite.

Un tel résultat est de peu d'importance, eu égard au nombre d'animaux en expérience, et ne dépasse même pas les survies aberrantes qui se peuvent manifester au cours de pareils essais.

II. Action du lait dans le rouget expérimental de la souris (1). - Nous partions d'une culture en bouillon Martin du bacille du rouget, mortelle pour la souris, en 48 à 60 heures, à la dose d'un cent millième de centimètre cube.

L'inoculation simultanée, mais en des points différents du corps, de cette dose mortelle et de $1 / 10 \mathrm{de}^{3} \mathrm{~cm}^{3}$ de lait stérilisé provoque la mort des souris ainsi inoculées en 48 heures.

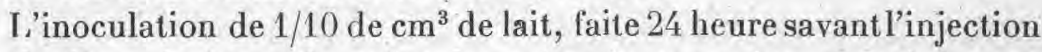
de la dose virulente de culture, amène la mort des organismes en 78 heures.

L'inoculation de la dose mortelle de culture, suivie, le lendemain et le surlendemain, d'une injection thérapeutique de $1 / 10 \mathrm{de} \mathrm{cm}^{3}$ de lait, causa la mort de ces souris en 84 heures.

Les résultats, ici, s'affirment et se précisent. La survie manifeste que les injections de lait, chez les souris atteintes expérimentalement du rouget, opposent à la mort, plaide en faveur de la méthode.

Alors que, dans le charbon du cobaye, la thérapeutique lactée se révèle impuissante, sinon à juguler, du moins à retarder la marche du processus morbide, il apparait que, dans le rouget de la souris, cette médication entraine un effort de l'organisme en vue de mieux s'armer et de se protéger contre l'infection envahissante. Sans doute les ressources organiques s'épuisent vite, puisque les indi-

(1) Nous sommes heureux de remercier un des travailleurs de notre laboratoire, M. KOLDA, qui nous a assistés dans cette partie de nos expériences, 
vidus traités succombent; il n'en est pas moins vrai que les injections de lait semblent accrồtre les défenses de l'économie et concourir d'une manière efficace à la protection des souris. Forts de ces résultats dogmatiques, nous avons voulu dès lors combattre différentes maladies par le moyen de cette "galactothérapie ». Qu'il s'agisse de septicémie hémorragique des bovidés, de diarrhée des veaux, de maladie des chiens, de diphtérie aviaire, nous n'avons enregistré que des échecs.

Au déclin d'une épizootie aphteuse des bovidés, la septicémie hémorragique vint se greffer sur les individus affaiblis, maigres, en mauvais état d'embonpoint.

Toutes les médications instituées ne purent empêcher l'évolution rapide d'une pasteurellose amenant la mort à brève échéance. Devant l'impuissance de la thérapeutique usuelle, nous essayâmes les injections inta-musculaires de lait bouilli répétées chaque matin, à la dose de $50 \mathrm{~cm}^{3}$ par animal et par jour. Ces inoculations furent effectuées sur 13 animaux ayant présenté dans les jours précédents des lésions aphteuses développées à la fois au niveau de la cavité buccale et des onglons.

La méthode se révéla aussi inefficace que les autres thérapeutiques; que la " galactothérapie " ait été pratiquée au début du processus morbide, au cours de son évolution ou à la période terminale, nulle influence ne parût s'exercer sur l'acuité, la durée et la marche de l'affection. Celle-ci se termina toujours, et à bref délai, par la mort des sujets frappés.

Il en fut exactement de même dans une épidémie de diarrhée des veaux où les injections quotidiennes de lait, effectuées à la dose de 20 à $25 \mathrm{~cm}^{3}$ par animal ne purent enrayer le flux diarrhéique et l'issue fatale $\mathrm{d}$ : la maladie. Une dizaine de veaux furent ainsi traités, lesquels étaient nés de vaches appartenant au foyer de fièvre aphteuse signalé précédemment.

Nos expériences furent pratiquées, sans aueun doute, dans de mauvaises conditions, puisque les animaux vivaient constamment dans un milieu richement infecté, où l'isolement étroit des malades était impossible et où la fièvre aphteuse sévissait en permanence. Cependant, il nous semble permis de conclure, mêmeen ces conditions, à l'inefficacité de la méthode ; en effet, nos animaux inoculés sont 
morts aussi bien et dans les mêmes délais que les individus qui ne bénéficiaient pas de cette thérapeutique.

La maladie du jeune âge, chez le chien, devait fournir un terrain exceptionnellement favorable à l'expérimentation. Nous n'avons pas manqué d'en profiter et nous avons essayé la valeur thérapeutique de la méthode dans les formes si diverses que peut revêtir la maladie des chiens, mais surtout dans ses localisations thoracique, abdominale et nerveuse (chorée, paraplégie).

Grâce à l'obligeance de notre collègue deshôpitaux, M.Lesbourriès, nous avons pu suivre plusieurs chiens atteints de localisations variées et quelquefois concomitantes de la maladie. Nos observations présentent de ce fait un certain intérêt, du point de vue spécial auquel nous nous sommes placés.

Nous injections systématiquement 5 à $10 \mathrm{~cm}^{3}$ de lait écrémé et stérile dans les masses musculaires de la fesse ou de la cuisse. La quantité de lait injectée était proportionnelle au poids de l'animal; suivant la gravitẻ du processus morbide, les inoculations étaient renouvelées chaque matin ou pratiquées seulement tous les deux jours.

a) Localisations thoraciques de la maladie du jeune áge. -3 cas, 3 échecs.

- La mort survint dans chaque cas comme si aucune médication n'avait été pratiquée. Le lait ne semble pas modifier l'état général de l'organisme, ni augmenter ses qualités de résistance contre les germes infectieux. Parallèlement, la médication lactée ne nous a procuré, en ces circonstances, aucune sédation des symptômes locaux : toux, jetage, essoufflement, lesquels gardent, chez les animaux traités, leurs caractères habituels. Le poumon ne parait pạs non plus bénéficier de l'injection lactée: aucune atténuation des râles ou du souffle tubaire, aucune diminution de la matité ne furent observées.

A l'autopsie des 3 sujets ainsi traités, nous avons effectué une dissection minutieuse des muscles de la fesse et de la cuisse. Cette exploration nous a permis de constater que le muscle résorbe d'une façon parfaite le lait écréıé et stérile, sans qu'il persiste au niveau des points d'inoculation la plus légère infiltration ou la moindre lésion de sclérose des fibres musculaires. 


\section{b) Localisations abdominales. -2 cas, 2 échecs.}

A vrai dire, un de ces animaux fait partie de la catégorie précédente : il présentait à la fois des signes de broncho-pneumonie et des symptômes graves d'entérite dysentérique.

Signalons en passant que, jusqu'à présent, l'administration per $o s$ d'iodure d'amidon - selon la technique proposée par le Docteur BRU, de Toulouse - nous a permis d'enregistrer des succès constants et inespérés dans le traitement des diarrhées - sanglantes ou non - des carnivores domestiques.

\section{c) Localisations nerveuses. -2 cas: 1 guérison, 1 échec.}

Le cas heureux, que nous signalons ici, n'est peut-être pas uniquement dû aux bons effets de la médication lactée. Il s'agissait d'un jeune fox, presque complètement paraplégique. Nous décidàmes d'associer, aux injections intra-musculaires de lait, les injections intra-veineuses d'urotropine (1). Il semble assez difficile de démêler la part qui revient au lait de celle qu'on peut rapporter à l'action interne de l'urotropine. Disons cependant qu'en ce qui nous concerne, et sur la foi d'autres observations, nous accorderions plus volontiers une action elficace et curatrice à l'urotropine.

\section{d) Localisations associées. - 2 cas, 2 échecs.}

Lorsque la maladie du jeune àge apparaît en même temps sur différents appareils, la médication lactée, dans les conditions de notre pratique, ne donne pas de résultats meilleurs. Cela peut, du reste, se concevoir, à prior $i$, à la lecture des faits exposés précédemment. La coexistence des formes nerveuse et pulmonaire ( 1 cas), ou nerveuse, pulmonaire et digestive ( 1 cas), crée dans les organismes atteints une dépression considérable; les injections parentérales de lait ne paraissent pas provoquer l'apparition de qualités nouvelles, antitoxique ou antiinfectieuse. L'intrication des symptômes, la complexité du processus morbide entrainent une déchéance plus rapide, plus accusée du sujet et la galactothérapie reste inopérante.

En résumé, dans toutes les localisations de la maladie des chiens où fut essayée la médication lactée, celle-ci se montra inefficace et impuissante à provoquer, par son emploi systématique, la résolution

(1) Nous nous proposons d'ailleurs de publier, dans un autre travail, les résultats acquis dans le traitement, par l'urotropine, des formes nerveuses de la maladie, 
des symptômes morbides, l'arrêt de la déchéance organique et un prompt retour vers l'état hygide antérieur.

Est-il besoin d'ajouter à tout cela l'inefficacité des injections de lait dans la diphtérie aviaire? Inoculé à la dose de $1 \mathrm{~cm}^{3}$ dans les muscles pectoraux, le lait ne provoqua aucune amélioration dans l'état général d'un coq et d'une poule diphtériques, non plus que la régression ou la disparition des membranes classiques. Celles-ci siégeaient sur le plancher buccal, à la face inférieure de la langue et autour de l'orifice de la trachée.

Que conclure de cette longue étude? Nous voulons et nous ne pouvons, par ces quelques essais, condamner une méthode - qui, entre les mains de certains auteurs - a donné d'excellents résultats. Nous nous sommes efforcés, dans la mesure de nos moyens, d'établir les indications pratiques de la galactothérapie. Nos expériences, réalisées en dehors de toute idée préconçue, à la fois sur des infections naturelles et sur des infections expérimentalement provoquées, n'ont abouti qu'à résultats décevants. Cependant, de notre pratique, nous gardons, très nette, l'impression que tout n'est pas dit en la matière et que, par une longue série d'études et de recherches systématiquement poursuivies, il sera possible depréciser les indications de la thérapeutique par le lait et de nous éclairer ainsi définitivement sur les avantages et la valeur de la méthode.

\section{LES MATIERES MINERALES DANS LE LAIT DE FEMME}

par le Prof. Dr Otakar LAXA

Directeur de l'Institut lactologique de l'Ecole polytechnique à Prague (Tchéco-Slovaquie).

Quoique les sels minéraux ne forment qu'une petite partie des matières solides dans le lait, leur importance est toutefois grande, d'abord au point de vue physiologique comme source des matières nécessaires à la construction du squelette du nouveau-né et ensuite au point vue technique comme substances régulatrices de l'action de la présure en fromagerie. C'est pourquoi il faut connaitre non seu- 